\title{
ESTUDO DE CROSSTALK EM PLACAS DE CIRCUITO IMPRESSO VIA KIT DIDÁTICO
}

\author{
CROSSTALK STUDY ON PRINTED CIRCUIT BOARDS THROUGH INSTRUCTIONAL KIT
}

Pablo Dutra da Silva ${ }^{1}$, Giovane Rodrigues de Oliveira ${ }^{2}$, Gustavo Melsi Floriani ${ }^{3}$

DOI: 10.37702/REE2236-0158.v39p400-411.2020

\begin{abstract}
RESUMO
Uma nova proposta de kit didático para estudo de crosstalk em Placas de Circuito Impresso (PCI) é apresentada no presente artigo. $\mathrm{O}$ referido kit tem diversos atributos que o tornam atraente para a utilização em cursos acadêmicos e em treinamentos dos mais variados níveis educacionais: do ensino técnico à pós-graduação. Entre os atributos citamos o baixo custo do kit, o baixo custo da estrutura laboratorial para a utilização deste, o pequeno peso e volume, colocando-o como opção para cursos de educação a distância. Além desses atributos físicos há os atributos didáticos, entre os quais está a possibilidade de estudo do crosstalk em PCI em dez diferentes arranjos de trilhas, proporcionando o estudo da influência da distância entre as trilhas, largura destas e do comprimento no problema de crosstalk de forma individualizada. Além disso, permite a análise de cada uma das situações com duas frequências de excitação diferentes dos arranjos, mantendo o tempo de transição entre níveis lógicos igual. Isso é uma característica positiva do ponto de vista didático, pois desmistifica a importância da frequência fundamental no projeto, visando à Compatibilidade Eletromagnética. A flexibilidade e possibilidades não terminam por aí, ainda há a possibilidade de excitar os arranjos com sinais externos ao kit e de testar medidas corretivas pós projeto como, por exemplo, a adição de capacitor para a redução da interferência. Essa proposta tem por objetivo apresentar uma solução para o contexto de raras atividades práticas em cursos da temática de Compatibilidade Eletromagnética, oportunizando atividades motivadoras e que proporcionam um aprendizado mais significativo. Os resultados apresentados demonstram que tanto o estudante quanto o professor têm a oportunidade de criar um ambiente de aprendizado que vai além da simples relação teoria-prática, desenvolvendo intuição nas melhores práticas de projeto de produtos eletrônicos.
\end{abstract}

Palavras-chave: Compatibilidade Eletromagnética; Crosstalk; Kit Didático; Placas de Circuito Impresso; Suporte a Aulas de Laboratório.

\begin{abstract}
A new proposal for an instructional kit for studying crosstalk on printed circuit boards is presented in this paper. This kit has several attributes that make it attractive for use in academic courses and training of various educational levels: from technical education to postgraduate. Among the attributes we mention the low cost of the kit, the low cost of the laboratory structure for its use, the small weight and volume, making it an option for distance education courses. In addition to these physical attributes there are the didactic attributes that are the possibility of studying crosstalk on printed circuit boards in ten different land arrangements providing insights about the influence of the distance between the lands, their width and length on the crosstalk problem individually. In addition, it allows the analysis of each situation with two different excitation frequencies keeping the same transition time between logical levels. This is a positive feature from a didactic point of view as it demystifies the importance of fundamental frequency in the project aiming at Electromagnetic Compatibility. The
\end{abstract}

\footnotetext{
${ }^{1}$ Docente da Engenharia Elétrica, Mestre em Engenharia Elétrica, Instituto Federal de Santa Catarina - Campus Rau, pablo.silva@ifsc.edu.br

${ }^{2}$ Acadêmico do Bacharelado em Engenharia Elétrica, Instituto Federal de Santa Catarina - Campus Rau, giovane.ro@aluno.ifsc.edu.br

3 Acadêmico do Bacharelado em Engenharia Elétrica, Instituto Federal de Santa Catarina - Campus Rau, gustavo.floriani1999@gmail.com
} 
flexibility and possibilities do not end there, there is still the possibility of exciting arrangements with external signals and testing post-project remedies, such as the addition of capacitor for interference reduction. This proposal aims to present a solution for the context of rare practical activities in Electromagnetic Compatibility courses providing motivating activities and meaningful learning. The results presented demonstrate that both the student and the teacher can create a learning environment that goes beyond the simple theory-practice relationship developing insights for the best design practices used in electronics design.

Keywords: Electromagnetic Compatibility; Crosstalk; Printed Circuit Board; Instructional kit; Laboratory Classes Support.

\section{INTRODUÇÃO}

A compatibilidade eletromagnética é a capacidade de um equipamento funcionar satisfatoriamente em seu ambiente eletromagnético, sem introduzir perturbação intolerável nos equipamentos que se encontrem nesse ambiente (IEC, 1990). Para isso, é necessário que os dispositivos eletrônicos não causem interferência em outros equipamentos, que sejam susceptíveis à interferência de outros sistemas - incluindo até mesmo organismos vivos -, e que não causem interferência em si mesmos (PAUL, 2016). Assim, será garantido o correto funcionamento dos equipamentos eletrônicos, evitando que interferências eletromagnéticas, na forma radiada, causem danos aos seres vivos. $\mathrm{O}$ ambiente eletromagnético está se tornando cada vez mais complexo; na indústria, em hospitais, nas residências e nos mais diversos locais há uma quantidade crescente de equipamentos eletrônicos que necessitam coabitar entre si e com os seres humanos (DEY; GHOSH, 2006).

Por conta dessa realidade, está se tornando de fundamental importância o desenvolvimento de habilidades relacionadas ou de projeto eletrônico considerando problemas de compatibilidade eletromagnética. $\mathrm{O}$ motivo para tal é que com o avanço tecnológico os usuários de sistemas eletrônicos exigem equipamentos com processamentos cada vez mais rápidos. Esse rápido processamento leva a circuitos que precisam ser mais velozes em sua resposta a estímulos de entrada e com frequências de clock cada vez maiores. Isso leva a circuitos com tempos de transição entre níveis lógicos cada vez menores, resultando em problemas de compatibilidade eletromagnética mais graves com relação à integridade de sinal (YIN et al., 2008), (CHENG et al., 2016).

O crosstalk, em Placa de Circuito Impresso (PCI), é também chamado diafonia na literatura brasileira e é definido como um acoplamento forte entre as trilhas de um circuito (QUESHI et al., 2017). Trata-se de um problema intrasistêmico que aumenta sua ocorrência com o avanço tecnológico de fabricação de PCI e o consequente adensamento do roteamento dos componentes (CHENG et al., 2016); (OUYANG; PHAN; XIAO, 2016). Essa interação entre trilhas da PCI pode acarretar distorções em sinais enviados, podendo causar erros de comunicação, redução da vida útil ou funcionamento incorreto de componentes. Identificar e prevenir o crosstalk é essencial para garantir o correto funcionamento dos equipamentos eletrônicos, tão presentes no cotidiano.

Desenvolver em alunos de graduação e de pós-graduação o conhecimento em projeto de Placas de Circuito Impresso, considerando problemas de compatibilidade eletromagnética, é fundamental para a formação de engenheiros eletricistas e eletrônicos no mundo contemporâneo. É necessário fazer com que o profissional engenheiro tenha a oportunidade de ter uma formação em que esse tema seja significativo, a fim de criar uma intuição com relação às suas práticas de projeto, resultando no desenvolvimento de projetos eletrônicos mais eficientes em todos os aspectos de um produto eletrônico (SILVA; OLIVEIRA; FLORIANI, 2018).

No contexto atual de Educação em Engenharia, especificamente no estudo de 
compatibilidade eletromagnética, são vistos poucos kits para estudos práticos. Utiliza-se, de forma mais abrangente, abordagens simuladas e teóricas (ADASCALITEI, 2006); (DEY; GHOSH, 2006). Além disso, os estudos têm se concentrado em propostas de simulação e estudo de maneiras de minimização estritamente no ambiente de pesquisa acadêmica (MUDAVATH; NAIK; GUGULOTHU, 2019); (XIAOSONG; RUNJING, 2007). Esses últimos têm pouco impacto na formação do engenheiro projetista em nível de graduação principalmente.

Dessa forma, projetistas de circuitos eletrônicos e acadêmicos das áreas de Engenharia Elétrica e Eletrônica devem estar capacitados para esse problema de compatibilidade eletromagnética. Entretanto, o crosstalk é visto na disciplina de Eletromagnetismo nos currículos das engenharias mencionadas, sendo pouco visto em outras disciplinas, como Eletrônica e Circuitos Elétricos. Com isso, a temática fica descontextualizada e menos significativa para os estudantes.

Assim, este artigo propõe um kit para estudo do crosstalk que poderá ser utilizado em unidades curriculares de graduação e pósgraduação, além de em treinamentos, a fim de tratar o tema com transversalidade e fomentar um processo educativo mais efetivo. $\mathrm{O}$ material didático poderá servir, também, para capacitar profissionais sobre a influência do crosstalk para a compatibilidade eletromagnética. $\mathrm{O}$ kit didático será de baixo custo, pequeno volume e peso, e os experimentos poderão ser realizados com osciloscópios habitualmente encontrados em instituições de ensino brasileiras, acarretando, portanto, baixo custo de infraestrutura para esses cursos. Além disso, sendo kits de baixo custo, estes têm a possibilidade de serem utilizados em disciplinas ou cursos de educação a distância (EaD), com o aluno utilizando os kits em casa.

Para que possamos entender como o kit poderá ser significativo, devemos entender quais são as oportunidades pedagógicas e de conteúdo que o estudante poderá ter e o professor ou instrutor poderá explorar para resultar no aprendizado significativo do tema. Para isso, faremos um resumo da teoria do crosstalk para o tipo de trilha construída no protótipo do kit. Na sequência faremos uma explanação da proposta de material didático e suas intencionalidades didáticas e, finalmente, demonstraremos como a teoria e os objetivos de aprendizagem se encontram na análise de algumas medidas feitas com o kit.

\section{CROSSTALK}

Segundo Paul (2016), o fenômeno de crosstalk acontece quando um sinal em um par de condutores aparece acoplado a um outro par de condutores adjacentes de forma indevida ou não intencional. Assim, como pode ser verificado no diagrama esquemático da Figura 1 (A), tomando três trilhas paralelas, temos uma trilha de retorno comum às outras duas, uma trilha agressora (aquela em que o sinal é enviado a um receptor de forma intencional) e a trilha vítima (adjacente à última, que será aquela que receberá a interferência). Além disso, podemos verificar o comprimento da trilha (Z). Já a Figura 1 (B) (MNAOUER; JAMEL; FETHI, 2010) apresenta a disposição das trilhas, um corte transversal à placa de circuito impresso com a apresentação das dimensões - largura da trilha (W), espaçamento entre trilhas (S) e espessura do dielétrico (h).

Figura 1 - Representação esquemática das trilhas e tensões $V_{\mathrm{NE}}, V_{\mathrm{FE}}$ e a dimensão de comprimento da trilha $(Z)$ na vista superior da PCI (A). Vista em corte transversal apresentando as dimensões largura $(\mathrm{W})$, espaçamento entre trilhas (S) e espessura do dielétrico (h) (B)

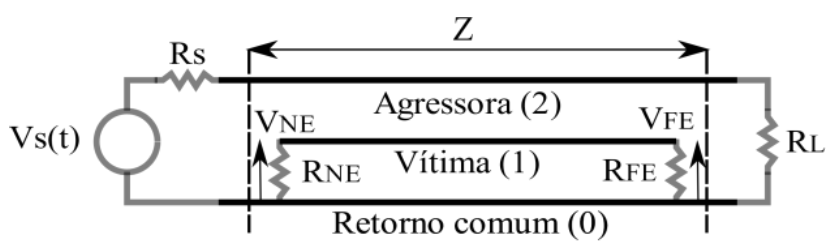

(A)

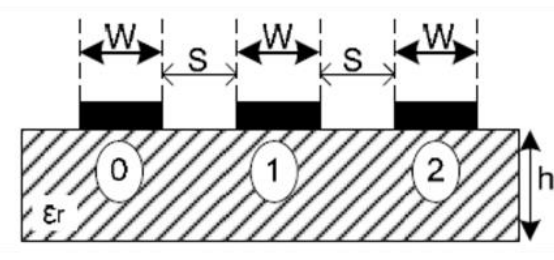

(B)

Fonte: acervo dos autores a partir de (MNAOUER; JAMEL; FETHI, 2010). 
Diversos trabalhos têm demonstrado que a interferência resultante na trilha vítima, que são representadas pelas tensões $V_{N E}$ e $V_{F E}$, têm influência tanto da tensão aplicada pelo sinal na trilha agressora quanto pela corrente que circula pelo circuito agressor (PAUL, 2016); (MNAOUER; JAMEL; FETHI, 2010); (YIN et al., 2008); (MUDAVATH; NAIK; GUGULOTHU, 2019); e (QUESHI et al., 2017). Dentro dos trabalhos citados verifica-se uma modelagem elétrica que representa o acoplamento por meio de uma indutância de acoplamento - (resultante da corrente) chamada indutância mútua $\left(L_{m}\right)$ e de uma capacitância de acoplamento - (resultante da tensão) - chamada de capacitância mútua $\left(C_{m}\right)$, conforme podemos ver nas equações (1) e (2) que representam $V_{N E}$ e $V_{F E}$ apresentadas por Paul (2016). Os demais modelos não serão apresentados por razões de escopo deste artigo, sendo o modelo apresentado apenas uma referência para o entendimento do fenômeno e de sua modelagem.

$$
\begin{aligned}
& =[\underbrace{\frac{R_{N E}}{R_{N E}+R_{F E}} L_{m} \frac{1}{R_{S}+R_{L}}}_{\text {Acoplamento Indutivo }}
\end{aligned}
$$

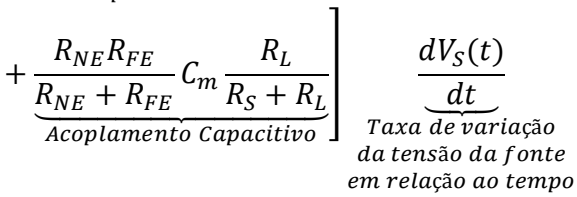

$$
\begin{aligned}
& =[\underbrace{V_{F E}(t)}_{\text {Acoplamento Indutivo }} \\
& +\frac{R_{F E}}{R_{N E}+R_{F E}} L_{m} \frac{1}{R_{S}+R_{L}} \\
& \underbrace{\frac{R_{N E} R_{F E}}{R_{N E}+R_{F E}} C_{m} \frac{R_{L}}{R_{S}+R_{L}}}_{\text {Acoplamento Capacitivo }}] \frac{d V_{S}(t)}{d t}
\end{aligned}
$$

Como $L_{m}$ e $C_{m}$ dependem basicamente das dimensões e de constantes físicas, mesmo não tendo uma equação explícita para esses valores é possível fazer uma análise qualitativa através de modelos mais simples. No caso da indutância esta dependerá da área composta pelas trilhas (1) e (0), apresentadas na Figura 1 (A), e do valor do campo magnético concatenado (abraçado) pela "espira" formada por este circuito e pelo valor da permeabilidade magnética (no caso específico a permeabilidade, $\mu_{r}$, é coincidente com a do ar por não haver materiais ferromagnéticos na estrutura). Já no caso da capacitância, esta dependerá das dimensões das trilhas, da permissividade elétrica do material dielétrico do substrato (este, no caso específico, é de uma permissividade $\varepsilon_{r}=6$ vezes a permissividade do ar) (PAUL, 2016); (MNAOUER; JAMEL; FETHI, 2010); (YIN et al., 2008); (MUDAVATH; NAIK; GUGULOTHU, 2019); e (QUESHI et al., 2017)

$\mathrm{Na}$ Figura 2 (A) podemos verificar um esquema didático para o entendimento da indutância mútua, em que é possível observar a geração de um campo magnético rotacional à corrente que o origina. Além disso, podemos observar a diferença da concentração das linhas de campo magnético à medida que se afastam da corrente originária. Considerando que a área da espira entre as trilhas (0) e (1) e o campo incidente nesta área formarão a base para o cálculo da indutância mútua segundo a equação (3) simplificada. Lembrando que esta valerá apenas para uma análise qualitativa simplificada.

$L=\frac{\mu}{2 \pi} Z \ln \left(\frac{\overbrace{2 W+2 S}^{\text {Distância entre as trilhas (2) } e(0)}}{\underbrace{S+W}_{\text {Distância entre as trilhas (2) } e(1)}}\right)$

Já na Figura 2 (B) podemos observar um esquema da configuração de campo elétrico simplificada que dará origem à capacitância mútua. Pode-se constatar, por exemplo, a concentração de linhas de campo elétrico maior dentro do substrato dielétrico devido ao efeito da permissividade maior do que a do ar. Para efeitos de análise qualitativa, consideraremos a equação (4) para o capacitor de placas planas, mas com a combinação dos efeitos de três capacitores formados pela trilha. $\mathrm{O} \alpha$ apresentado na equação (4) é referente à espessura do cobre da trilha. 


$$
\begin{aligned}
C=\underbrace{\varepsilon_{0} \frac{W \cdot Z}{S+W}}_{\begin{array}{c}
\text { Dielétrico ar } \\
\text { face superior } \\
\text { das trilhas }
\end{array}}+\underbrace{\varepsilon_{0} \frac{Z . \alpha}{S}}_{\begin{array}{c}
\text { Dielétrico ar } \\
\text { Face interior } \\
\text { entre as trilhas }
\end{array}} \\
+\underbrace{\varepsilon \frac{W \cdot Z}{S+W}}_{\begin{array}{r}
\text { Dielétrico } \\
\text { Face inferior } \\
\text { das trilhas }
\end{array}}
\end{aligned}
$$

Figura 2 - Representação Esquemática do campo magnético quando a trilha (2) é percorrida por corrente no sentido entrando no plano da folha (A) e do campo elétrico devido a uma diferença de potencial entre as trilhas (1) e (2) (B)

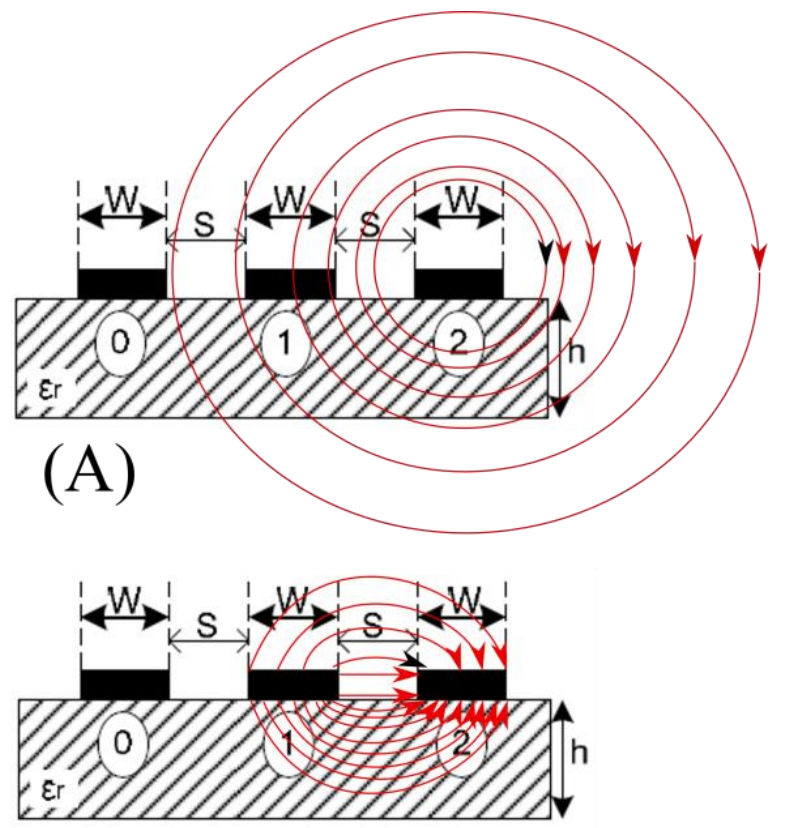

(B)

Fonte: acervo dos autores.

Então, as tensões $V_{N E}$ e $V_{F E}$, modeladas pelas equações (1) $\mathrm{E}(2)$, tendem a diminuir à medida que o espaçamento $S$ entre as trilhas aumenta, pois como pode ser observado nas equações (3) E (4) $L_{m}$ não muda seu valor significativamente, mas $C_{m}$ sim. Este é reduzido de forma inversamente proporcional ao aumento de S. Uma segunda observação que podemos fazer das equações (1), (2), (3) e (4) é que tanto $L_{m}$ quanto $C_{m}$ são diretamente proporcionais ao comprimento das trilhas $(\mathrm{Z})$, fazendo com que se aumente o acoplamento entre elas. Ou seja, quanto maior a extensão adjacente entras as trilhas agressora e vítima maior o acoplamento entre elas. A terceira observação relacionada às dimensões da estrutura física do leiaute da placa de circuito impresso tem relação com a influência de W, pode-se verificar nas equações (1), (2), (3) e (4) que esta dimensão não causa influência significativa com relação à $L_{m}$ e $C_{m}$, já que em ambos há uma proporcionalidade tanto direta quanto inversa, causando muito pouco impacto no acoplamento (PAUL, 2016); (MNAOUER; JAMEL; FETHI, 2010); (YIN et al., 2008); (MUDAVATH; NAIK; GUGULOTHU, 2019) e (QUESHI et al., 2017)

As resistências $R_{N E}$ e $R_{F E}$, bem como $R_{L}$, também têm influência sobre as tensões $V_{N E} \mathrm{e}$ $V_{F E}$, segundo as equações (1) e (2). Para esta proposta de kit optamos por considerá-las com o mesmo valor de $1 k \Omega$ para ser analisadas apenas as influências das três variáveis mencionadas anteriormente. Outro efeito dessa escolha é que a influência da capacitância fica mais evidente em relação à influência da indutância. Essa escolha também se deu por ordem prática, pois as impedâncias típicas das entradas de circuitos digitais e de sinais mistos está nesta ordem de grandeza. Dependendo da tecnologia empregada na fabricação desses circuitos, as impedâncias são ainda maiores, diferentemente da literatura didática (PAUL, 2016) e da literatura científica (MNAOUER; JAMEL; FETHI, 2010); (YIN et al., 2008); (MUDAVATH; NAIK; GUGULOTHU, 2019); e (QUESHI et al., 2017), que utilizam geradores e cargas de rádio frequência (tipicamente $50 \Omega$ ) para fazer casamento de impedâncias. Na prática, a impedância utilizada evidencia mais os problemas enfrentados em crosstalk no caso de se medir com um osciloscópio de bancada de uso geral (SILVA; OLIVEIRA; FLORIANI, 2018).

A última influência importantíssima, além das características construtivas e de carga já mencionadas, é a característica do sinal que é aplicado à trilha agressora. Essa também influencia drasticamente no problema de integridade de sinal já apresentado em Silva, Oliveira e Floriani (2018). A taxa de variação do sinal aplicado à trilha agressora $\left(d V_{S}(t) / d t\right)$ é diretamente proporcional às tensões de crosstalk $V_{N E}$ e $V_{F E}$. Isso pode ser verificado nas equações (1) e (2). É importante salientar que essa taxa de variação não está diretamente ligada à frequência fundamental do sinal aplicado, mas sim às mudanças repentinas de 
tensão. Isto é mais bem verificado em sinais do tipo retangular, pois, mesmo apresentando baixíssima frequência fundamental, pode ter um tempo de transição entre níveis muito rápido, ocasionando assim problemas tanto de integridade quanto de crosstalk (PAUL, 2016); (SILVA; OLIVEIRA; FLORIANI, 2018).

No caso de integridade de sinal, estudado em Silva, Oliveira e Floriani (2018), a relação entre o tempo de propagação da linha de transmissão formada pela trilha agressora é relevante e deve ser comparada com o tempo de transição do sinal aplicado, ou seja, $d V_{S}(t) / d t$ (PAUL, 2016); (SILVA; OLIVEIRA; FLORIANI, 2018). No caso do crosstalk não há relevância com relação $d V_{S}(t) / d t$ a essa comparação, como tivemos a oportunidade de verificar durante este estudo. No entanto, podemos verificar que, assim como em integridade de sinal, o tempo de transição entre níveis lógicos também deve ser considerado, mas em seu valor absoluto e não em comparação a algum outro. Isso não deve ser confundido com a frequência fundamental, o que podemos afirmar que é uma confusão bastante comum entre projetistas, pois, como verificaremos nos resultados deste artigo, mesmo com baixas frequências, mas com o $d V_{S}(t) / d t$ mantido com relação a um sinal de alta frequência os valores das tensões $V_{N E} \mathrm{e}$ $V_{F E}$ se mantêm inalterados. Isso pode ser observado também em (SILVA; OLIVEIRA; FLORIANI, 2018) para o caso de integridade de sinal em linhas de transmissão.

\section{PROPOSTA DE MATERIAL DIDÁTICO E SUA INTENSIONALISADES}

O material didático é composto de trilhas de circuito impresso em placa de fibra de vidro. O sinal para verificação dos resultados será originado por um circuito integrado o qual gerará um sinal do tipo retangular com sua frequência ajustável em $32 \mathrm{kHz}$ ou $4 \mathrm{MHz}$ com tempo de transição de $5 \mathrm{~ns}$ para ambas as frequências. Essa característica mantém o $d V_{S}(t) / d t$ do sinal igual para ambas as frequências e garante que o aluno poderá perceber a questão da importância de se considerar o tempo de transição, ou o $d V_{S}(t) /$ $d t$, e não apenas a frequência fundamental do sinal, assim como foi feito em Silva, Oliveira e Floriani (2018) para o problema de integridade de sinal em trilhas de placas de circuito impresso. Isso possibilitará o estudo da influência desse parâmetro para o aparecimento e minimização do crosstalk. Além do que, ajudará a desmitificar a crença da frequência fundamental como a única consideração a ser feita e que essa, em alguns casos, pode levar a erros grosseiros.

Para estudo da influência dos parâmetros construtivos das trilhas será oferecido um total de 10 conjuntos de três trilhas cada. Cada conjunto simula o arranjo esquemático apresentado na Figura 1 (A), em que há uma trilha de retorno do sinal comum para as trilhas agressora e vítima; a trilha vítima se localiza entre a trilha agressora e a trilha de retorno, e a trilha agressora é uma das mais externas. Cada um desses conjuntos tem como carga da trilha vítima resistores de $1 \mathrm{k} \Omega$ no início e no fim da trilha, e a trilha agressora conta com uma carga resistiva de mesmo valor cujas razões para esta escolha já foram apresentadas na seção anterior. A Figura 1 (A) apresenta o esquema de ligação dessas resistências e a ligação da fonte $V_{S}(t)$ já descrita anteriormente. Cada um desses 10 conjuntos pode ser analisado separadamente e tem dispositivos para isolar um conjunto do outro e o sinal gerado para o teste, o que possibilita até, segundo a intencionalidade do professor que estiver utilizando, a aplicação de sinais externos. Isso poderia acontecer para reforçar o aprendizado dos cuidados necessários com relação às características deste anteriormente mencionadas. Existe também a possibilidade de inserção de componentes, como por exemplo o resistor de casamento de impedâncias ou de capacitores de filtro por parte do professor, para que se possa apresentar técnicas de minimização do problema de forma prática. É importante salientar que esse tipo de técnica é largamente utilizada na indústria eletrônica, apesar de todos os esforços no sentido de se fazer um projeto eletromagneticamente compatível, e que também deve ser de conhecimento do estudante como aplicá-los. 
Os 10 conjuntos, já mencionados, têm por objetivo didático oferecer estruturas físicas com duas características construtivas, $\mathrm{S}, \mathrm{W}$ e $\mathrm{Z}$ iguais e com apenas uma delas diferente. Isso para que seja possível isolar as influências dessas diferentes características e conectá-las, individualmente, à severidade do problema. Há quatro conjuntos em que os valores de $\mathrm{Z}$ e de $\mathrm{W}$ se mantêm constantes e o estudante poderá verificar a influência apenas de $\mathrm{S}$ na severidade do problema de crosstalk na trilha vítima. Há também três conjuntos em que tanto $S$ quanto $Z$ se mantêm constantes, mas $\mathrm{W}$ apresenta diferentes valores para que o estudante possa verificar a influência deste. Mais três conjuntos utilizam a mesma estratégia para o estudo da influência do comprimento da trilha $\mathrm{Z}$.

A alimentação do sistema é realizada através de fonte de alimentação em corrente contínua, comum em bancadas de ensino de eletrônica, ou por porta USB, caso se opte por este tipo de conector, com tensão de 2,7 à 5,5 V . Para fins de representação de resultados, neste trabalho optou-se pela tensão de $5 V$. Assim, as trilhas receberão o sinal enviado pelo gerador de sinal. Para ajustar a frequência, utiliza-se uma chave seletora que muda a frequência do sinal entre dois valores: $4 \mathrm{MHz}$ e $32 \mathrm{kHz}$. É interessante lembrar que o tempo de transição entre os níveis do sinal gerado se mantém inalterado para quaisquer das situações de alimentação e de frequência gerada aqui descritas. Isso é de fundamental importância para os objetivos didáticos aqui apresentados.

Para a verificação do crosstalk entre trilhas, é necessário medir a tensão induzida entre as trilhas agressora e vítima, sendo estas $V_{N E}$ e $V_{F E}$. Essas tensões aparecem sobre os resistores $R_{N E}$ e $R_{F E}$, respectivamente. Nessas tensões o prefixo $N E$ e $F E$ representam near end, ou "extremidade próxima", e far end, ou "extremidade distante", respectivamente em relação ao gerador do sinal (PAUL, 2016). De forma análoga, a tensão $V_{R L}$ representa a tensão sobre o resistor da carga $R_{L}$. A verificação do efeito do acoplamento entre as trilhas é aferida apenas com a medida das tensões $V_{N E}$ e $V_{F E}$. Isso é realizado com o auxílio de um osciloscópio o qual, para medir a tensão $V_{N E}$, deve ter suas ponteiras conectadas nos terminais do resistor $R_{N E}$. Analogamente, para medir a tensão $V_{F E}$, coloca-se as ponteiras do osciloscópio em $R_{F E}$.

Os resultados devem sempre ser analisados de forma comparativa, pois sempre há algum nível de interferência. O que se deve comparar, para uma análise, é o quanto uma situação agrava ou minimiza a interferência que se apresenta em todos os conjuntos. O professor ou o estudante estará livre para definir um método para realizar tal comparação, pois o kit permitirá essa liberdade. Mas, sugere-se aferir uma das tensões, registrar em um pen-drive no osciloscópio e depois registrar a outra tensão, evitando assim a interferência entre medições. Ao final de todas as medições, salvas em um pen-drive, com o osciloscópio, é possível desenhar em um mesmo gráfico todas as curvas com a utilização de um software matemático. A diferença entre os picos de tensão induzida, ao analisar uma mesma variável $(\mathrm{S}, \mathrm{W}, \mathrm{Z}$ ou $\left.d V_{S}(t) / d t\right)$, representa a sua contribuição para a minimização ou agravamento do crosstalk. A aquisição dos dados via osciloscópio digital permitirá a comparação com simulação de circuitos de diversos modelos apresentados na literatura, como exemplo em Paul (2016), Yin et al. (2008), Jing e Xiasong (2007), Novak (1994), Han (2006) e Mnaouer, Jamel e Fethi (2010). Alguns exemplos de possíveis análises de dados utilizando o kit poderão ser verificados na seção de resultados deste artigo. Podemos verificar o quanto é didática a análise dos resultados quando estes estão compilados em um único gráfico, pois, além de apresentar ao estudante $o$ formato típico de uma interferência por crosstalk para futuras identificações qualitativas nos problemas reais, ela mostra o quanto uma característica afeta a gravidade do problema.

Os autores pedem escusas por não apresentar alguma figura que mostre as trilhas construídas; no entanto, isso se faz necessário devido ao processo de depósito de patente a que este trabalho está sendo submetido. Apresentamos alguns valores de referência de medidas que estão sendo utilizadas para a posterior análise dos resultados. Como já mencionado, há quatro conjuntos de trilhas para estudo da influência de $\mathrm{S}$, sendo utilizados os valores 20, 50, 100 e 200 mils, mantendo-se as 
demais características físicas inalteradas entre os conjuntos. Para teste da influência de $W$, há quatro conjuntos de trilhas com os valores 20 , 35, 50 e 100 mils, mantendo-se as demais variáveis constantes. Para verificação da influência de $Z$, há três conjuntos de trilhas de 5,15 e $30 \mathrm{~cm}$ de comprimento, mantendo-se as demais variáveis constantes.

\section{RESULTADOS E ANÁLISES}

A fim de verificar a influência do espaçamento $S$ entre as trilhas, manteve-se as demais variáveis constantes. Utilizando valores de $S=20,50$ e 100 e 200 mils , já mencionados, mas repetidos aqui por conveniência, o kit permite observar os picos das tensões $V_{N E}$ e $V_{F E}$ (na trilha vítima) aumentarem com a redução do espaçamento $S$. A demonstração dos picos de $V_{F E}$ para $S=$ 20,50 e 100 mils estão representadas na Figura 3. A frequência do sinal aplicado à trilha agressora é de $4 \mathrm{MHz}$ com tempo de transição de 5 ns e tensão final de $5 \mathrm{~V}$.

Figura 3 - Influência do espaçamento $S$ entre as trilhas na tensão VFE

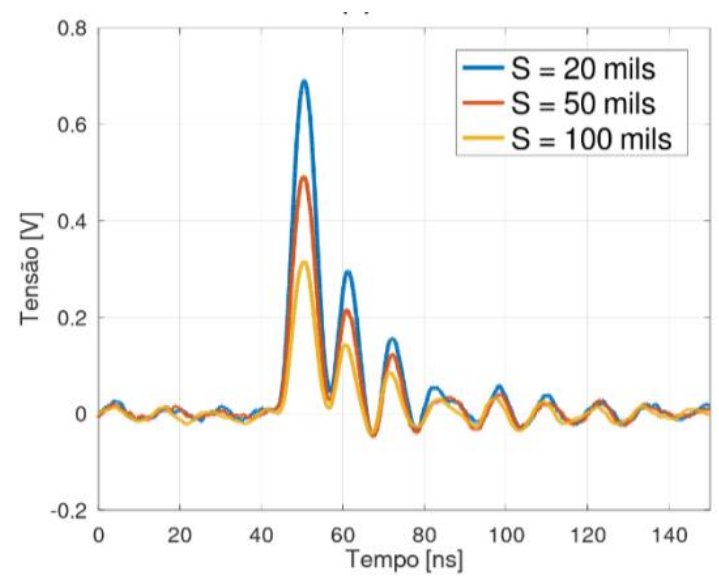

Fonte: acervo dos autores.

A Figura 4 apresenta a tensão $V_{\mathrm{NE}}$ para as mesmas trilhas utilizadas para a medição dos dados apresentados na Figura 3. Pode-se verificar o formato muito similar nas formas de onda com relação às medidas de $\mathrm{V}_{\mathrm{FE}}$. Outra observação importante é que o valor de pico é maior em relação ao valor máximo da interferência em $\mathrm{V}_{\mathrm{NE}}$. Essa diferença pode ser explicada pelas equações (1) e (2), em que para
VFE uma das parcelas é negativa, fazendo com que o valor seja menor do que o valor de $\mathrm{V}_{\mathrm{NE}}$.

Figura 4 - Influência do espaçamento $S$ entre as rilhas na tensão $V_{N E}$

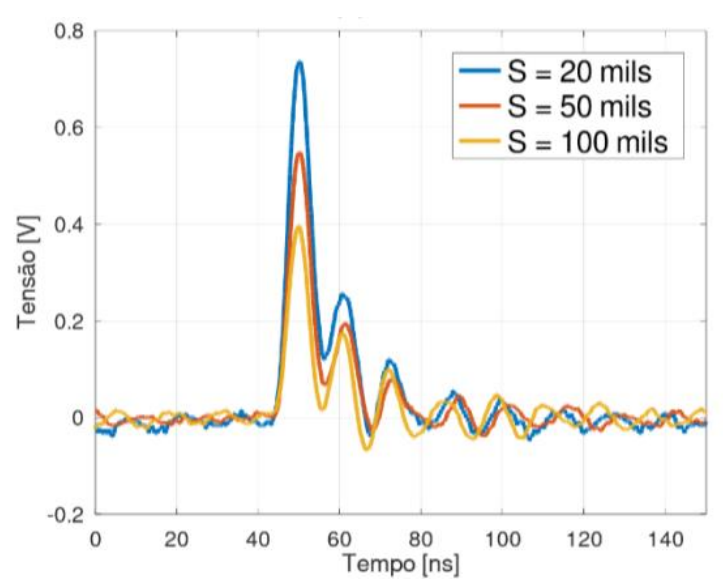

Fonte: acervo dos autores.

Verificando a Figura 3, Figura 4 e Figura 5 , podemos observar a influência de $S$, comparando as respostas dos valores medidos de $V_{F E}$ e $V_{N E}$, para uma excitação de $4 \mathrm{MHz}$, e $V_{N E}$, para uma excitação de $32 \mathrm{kHz}$, respectivamente. Conclui-se que para qualquer situação, quanto maior o $\mathrm{S}$ menor será a tensão de crosstalk. O estudante pode analisar as razões para isso nas equações (1), (2), (3) e (4). $\mathrm{Na}$ equação (3), verifica-se que a indutância tende a se manter a mesma enquanto a capacitância é reduzida. Na equação (1), verifica-se a proporcionalidade direta com a capacitância mútua - conforme ainda pode ser verificado comparando-se a Figura 4 e a Figura 5 , que diferem apenas na frequência do sinal que é aplicado à trilha agressora - em que os resultados diferem muito pouco com relação à mudança da frequência. Isso porque para ambas as situações o $d V_{S}(t) / d t$ se mantém praticamente o mesmo. Podemos demonstrar para o estudante, realizando esse cruzamento de informações, que a frequência fundamental não é a característica principal a ser considerada, mas $\operatorname{sim}$ o $d V_{S}(t) / d t$ que se mantém inalterado no kit apresentado. Todas essas percepções podem ser estudadas na primeira parte do kit.

Na continuidade o estudante pode estudar a influência do $\mathrm{W}=20,50$ e 100 mils na tensão $V_{F E}$ de crosstalk. A Figura 6 apresenta os resultados das medidas de $V_{F E}$ para os valores 
de $\mathrm{W}$ especificados nela. $\mathrm{O}$ estudante pode perceber que o aumento de $\mathrm{W}$ mantém a capacitância e as indutâncias inalteradas, concluindo que a tensão de crosstalk não serão alteradas com a alteração apenas desse parâmetro. Tal resultado pode ser verificado também nas equações (3) e (4).

Figura 5 - Resultados das medidas de $\mathrm{V}_{\mathrm{NE}}$ com uma frequência de $32 \mathrm{kHz}$ aplicado à trilha agressora

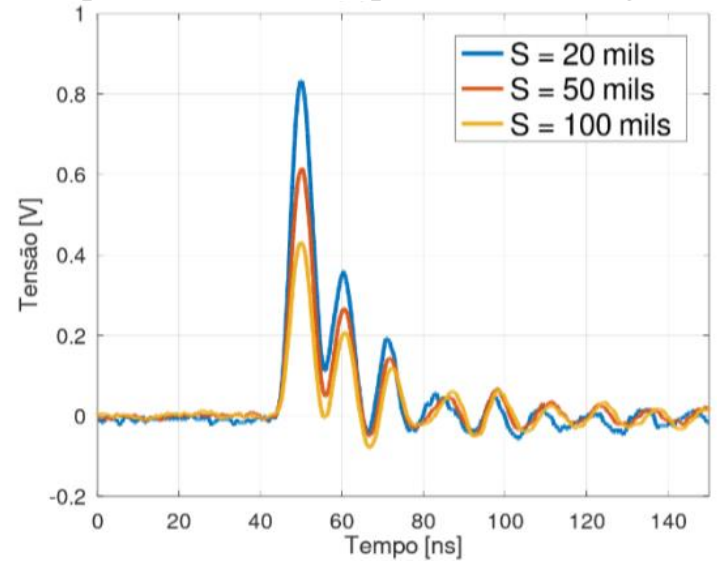

Fonte: acervo dos autores.

Além disso, pode-se verificar o comportamento de $V_{F E}$ e o comportamento com a frequência (resultados não apresentados aqui, pois levam às conclusões já tiradas durante o estudo de $\mathrm{S}$ ). Com os resultados observados o estudante verifica que o $\mathrm{W}$ não é um parâmetro tão importante no processo quanto $\mathrm{S}$, já que o último apresenta uma influência mais latente e que $\mathrm{W}$ é uma característica, que na prática, se mantém a menor possível com relação à corrente que circulará nessa trilha por razões da densidade de roteamento. Ou seja, trilhas mais grossas do que o mínimo necessário ocupam um espaço desnecessário.

Figura 6 - Influência da espessura $W$ das trilhas na tensão $V_{\mathrm{NE}}$

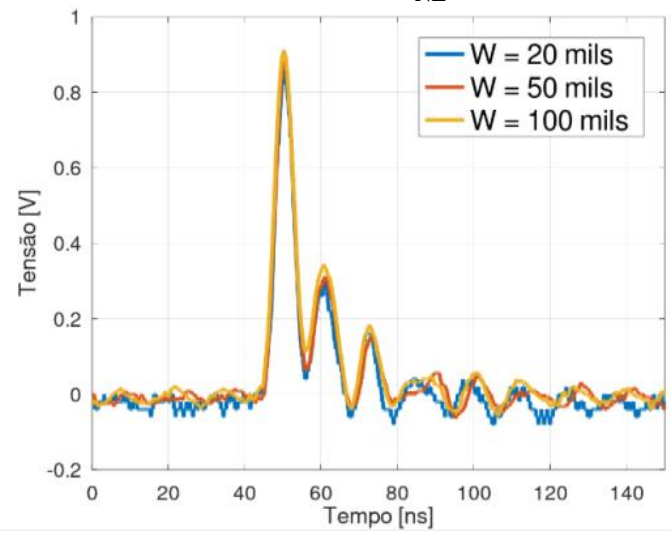

Fonte: acervo dos autores.
Já para a influência do comprimento $Z$, apresentada na Figura 7 , os resultados se apresentaram consistentes com as equações (3) e (4), ou seja, tendo valores de tensão $V_{F E}$ diretamente proporcionais ao aumento do comprimento $Z$ das trilhas. Isso tanto para a capacitância mútua quanto para a indutância. A demonstração dos picos de $V_{N E}$ para $Z=$ 5,15 e $30 \mathrm{~cm}$ demonstram exatamente isso. Os resultados apresentados demonstram para $\mathrm{o}$ estudante o quanto essa variável é importante na redução do crosstalk. É necessário evitar a construção de trilhas paralelas àquelas que são alimentadas por sinais de $d V_{S}(t) / d t$ altos.

Nas Figura 3, Figura 6 e Figura 7, pôde-se verificar a convergência dos resultados de medição com a teoria apresentada. Os resultados para $V_{N E}$ também seguiram a mesma tendência apresentada na Figura 4, convergindo para a teoria e mantendo valores nitidamente diferentes quando se alterava as variáveis. Trabalhando na frequência de $32 \mathrm{kHz}$, os demais resultados convergiram para a teoria e para a análise realizada para a Figura 5, por isso optamos por não repetir os resultados para as demais situações, pois resultam em análises desnecessárias.

Figura 7 - Influência do espaçamento $Z$ entre as trilhas na tensão $V_{F E}$

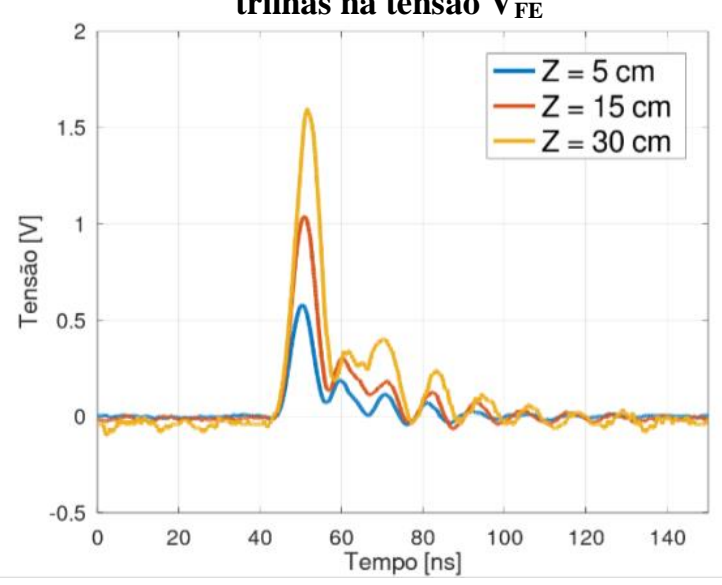

Fonte: acervo dos autores.

Conforme já foi mencionado anteriormente, o kit permitirá o teste de maneiras de minimizar o problema caso as demais características físicas das trilhas estudadas não possam ser mudadas. Uma das maneiras mais comuns de realizar isso é a adição de um capacitor de filtro em paralelo com a carga nas pontas da trilha vítima. Para o 
teste adicionou-se um capacitor de $47 \mathrm{nF}$ na ponta da tensão $V_{N E}$ para a trilha de $Z=15 \mathrm{~cm}$ e foi possível verificar a drástica redução da interferência de crosstalk nesse arranjo de trilhas testado. É importante salientar que tal resultado se repete para os demais arranjos.

Figura 8 - Comparação da interferência de crosstalk com e sem a utilização de um capacitor de filtro na ponta da trilha vítima

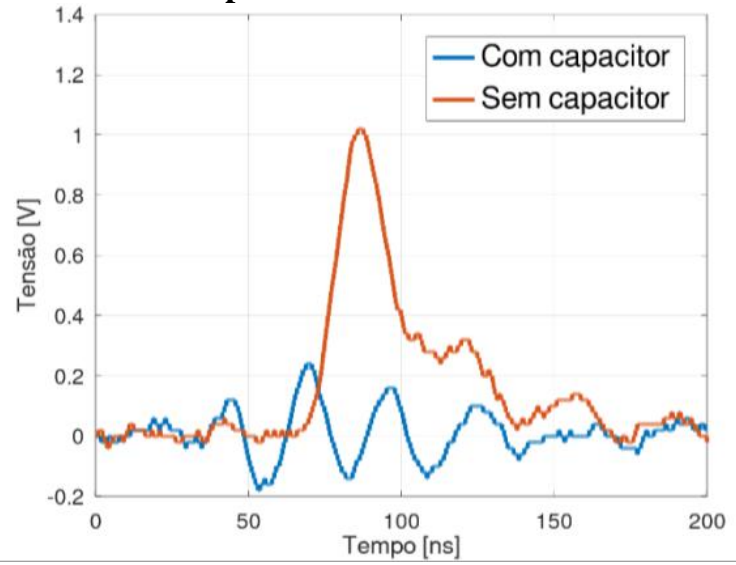

Fonte: acervo dos autores.

\section{CONSIDERAÇÕES FINAIS}

Após análise dos possíveis resultados que o kit pode proporcionar ao estudante e ao professor que o utilizar, podemos concluir que o material didático mostra claramente a influência da distância $S$ entre trilhas, espessura $W$ das trilhas e seus comprimentos $Z$ para as tensões $V_{N E}$ e $V_{F E}$. Ademais, além de oportunizar o estudo prático da influência das citadas características físicas das trilhas nas tensões induzidas em uma trilha vítima, o kit também oportuniza o estudo de maneiras de minimizar o problema. Dessa forma, o kit didático se apresenta como excelente material para estudo do acoplamento forte entre trilhas de PCI, sendo objeto de baixo custo que vem a somar às capacitações de acadêmicos de Engenharia Elétrica e Eletrônica.

As medidas apresentadas foram realizadas com osciloscópios de baixo custo, amplamente encontrados em laboratórios de estudo de eletrônica de instituições de todo o país, mostrando-se, assim, como uma excelente oportunidade de oferecer estudo prático de problemas de compatibilidade eletromagnética a baixo custo, assim como o kit já apresentado no Congresso Brasileiro de Educação em Engenharia - COBENGE 2018, pelos mesmos autores deste artigo, este com patente já depositada. Vale salientar que esse kit anterior serve para o estudo de outros problemas de compatibilidade eletromagnética em Placas de Circuito Impresso e de propagação e ondas em linhas de transmissão.

Concluímos que o kit desenvolvido por esta equipe se mostra confiável e adequado para a tarefa de atender às demandas por atividades práticas nos cursos de Compatibilidade Eletromagnética, demandas que se relacionam à formação de Engenheiros com raciocínio analítico e sintético com relação às problemáticas levantadas pelo kit. Ainda, tal kit aguça a intuição e repertório prático para os projetos de Placas de Circuito Impresso considerando os problemas de compatibilidade eletromagnética. Sempre é válido lembrar que profissionais com as habilidades citadas são muito bem-vindos no mercado. Além do mais, o uso do kit e a realização de atividades práticas tornam os cursos mais interessantes, motivadores e permitem um aprendizado mais significativo.

\section{AGRADECIMENTOS}

Os autores agradecem o apoio financeiro dado ao projeto pelo Instituto Federal de Santa Catariana, por intermédio do Edital Universal $\mathrm{N}^{\circ} 02 / 2018$ e da chamada interna $\mathrm{N}^{\mathrm{o}} 03 / 2018$.

\section{REFERÊNCIAS}

ADASCALITEI, A. A. Courseware Structure For Online Electromagnetic Compatibility. 2006. IEEE International Symposium on Industrial Electronics, p. 3210-3213, Montreal, Que., 2006.

CHENG, M-H. et al. Microstrip lines far-end crosstalk cancellation using striplines in hybrid PCB structure. 2016. Asia-Pacific International Symposium on Electromagnetic Compatibility (APEMC), p. 576-579, Shenzhen, 2016. 
DEY, T. K.; GHOSH, C. K. Proposed methodology of EMC teaching through Distance Education. 2006. 9th International Conference on Electromagnetic Interference and Compatibility (INCEMIC 2006), p. 93-97, Bangalore, 2006.

HAN, J. K.; PARK, D. C. Crosstalk Analysis Between Non-Parallel Coupled Lines Connected With Vias in a 4-layer PCB. 2006. 4th Asia-Pacific Conference on Environmental Electromagnetics, p. 728-731, Dalian, 2006.

IEC 60050-161. International electrotechnical vocabular, chapter 161: Electromagnetic compatibility, International Electrotechnical Comission (IEC), Geneva, Standard, 1990.

MNAOUER, K.; JAMEL, B. H. T.; FETHI, C. Modeling of microstrip and PCB traces to enhance crosstalk reduction. 2010. IEEE Region 8 International Conference on Computational Technologies in Electrical and Electronics Engineering (SIBIRCON), Listvyanka, 2010, pp. 594-597.

MUDAVATH, R.; NAIK, B. R.; GUGULOTHU, B. Analysis of Crosstalk Noise for Coupled Microstrip Interconnect Models in HighSpeed PCB Design. 2019. International Conference on Electronics, Information, and Communication (ICEIC), p. 1-5, Auckland, New Zealand, 2019.

QUESHI, N. et al. Strong coupling (crosstalk) between printed microstrip transmission lands on printed circuit boards. 2017. 14th International Bhurban Conference on Applied Sciences and Technology (IBCAST), p. 711716, 2017.

NOVAK, I.; EGED, B.; HATVANI, L. Measurement and simulation of crosstalk reduction by discrete discontinuities along coupled PCB traces. IEEE Transactions on Instrumentation and Measurement, v. 43, n. 2, p. 170-175, April, 1994.

OUYANG, G.; PHAN, L. T.; XIAO K. Methods to reduce crosstalk in flex circuit and $\mathrm{PCB}$. 2016. IEEE International Symposium on Electromagnetic Compatibility (EMC), p. 2528, Ottawa, ON, 2016.
PAUL, C. R. Eletromagnetismo para Engenheiros: com aplicações a sistemas digitais e interferência eletromagnética. Rio de Janeiro: LTC, 2016.

SILVA, P. D.; OLIVEIRA, G. R.; FLORIANI, G. $M$. Proposta de kit didático para o estudo de integridade de sinal em placas de circuito impresso. 2018. Congresso Brasileiro de Educação em Engenharia (COBENGE), Salvador, 2018.

TANI, L.; OUAZZANI, N. E. Minimizing crosstalk on printed circuit board using non uniform guard traces. 2016. International Conference on Information Technology for Organizations Development (IT4OD), p. 1-4, Fez, 2016.

TIAN, W. et al. Optimized design of signal crosstalk in high speed PCB. 2012. 13th International Conference on Electronic Packaging Technology \& High Density Packaging, p. 549-551, Guilin, 2012.

WU, J. et al. The crosstalk analysis of packagePCB complex interconnect structure. 2017. IEEE Electrical Design of Advanced Packaging and Systems Symposium (EDAPS), p. 1-3, Haining, 2017.

XIAOSONG, J.; RUNJING, Z. Crosstalk Analysis and Simulation in High-Speed PCB Design. 2007. 8th International Conference on Electronic Measurement and Instruments, p. 2437-2440, Xi'an, 2007.

YIN, Q. et al. Analysis of crosstalk in PCB design. 2008. 8th International Symposium on Antennas, Propagation and EM Theory, p. 1071-1074, Kunming, 2008. 


\section{DADOS BIOGRÁFICOS DOS AUTORES}

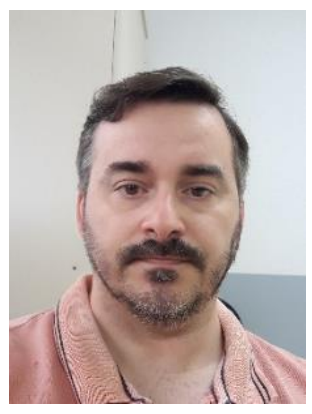

Pablo Dutra da Silva - Graduado em Engenharia Elétrica pela Universidade Federal de Santa Catarina (UFSC) em 2004. Mestre em Engenharia Elétrica, área de concentração circuitos e sistemas integrados, na UFSC em 2006. Atua como Professor no Instituto Federal de Santa Catarina desde 2015, lecionando para cursos técnicos e atualmente com dedicação ao curso de Bacharelado em Engenharia Elétrica do Campus Jaraguá do Sul - Rau. Em 2017 terminou na 2a colocação no Prêmio IFSC de Inovação na categoria sala de aula pelo trabalho realizado na Unidade Curricular de Projeto Integrador I - Iniciação Científica, e em 2019 recebeu junto aos seus orientandos um aporte financeiro para a realização de projeto de inovação tecnológica por ter terminado o Prêmio IFSC de ideias inovadoras entre as oito melhores equipes. Trabalhou no desenvolvimento de produtos eletrônicos por sete anos entre indústria de semicondutores e de eletrônicos em geral. Atua como docente há, aproximadamente, seis anos; quase todos com o Ensino de Engenharia, área em que atua com educação baseada em projetos e outras metodologias ativas de ensino e com avaliação processual.

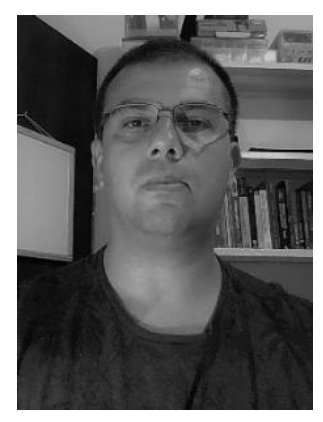

Giovane Rodrigues de Oliveira - Atualmente é graduando em Engenharia Elétrica, pela Instituto Federal de Santa Catarina no Campus Jaraguá do Sul - Rau. Realizou pesquisa na área de Eletrônica para elaboração de kit para estudo de compatibilidade eletromagnética. Além disso, atuou como monitor de Cálculo e Álgebra Linear e no projeto de extensão, pesquisando potencialidades para extensão na região de Jaraguá do Sul - SC. Assuntos de interesse: compatibilidade eletromagnética, crosstalk, integridade de sinal.

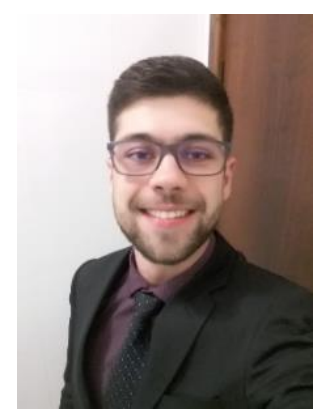

Gustavo Melsi Floriani - Atualmente é graduando em Engenharia de Computação, pelo Instituto Federal Catarinense no Campus São Bento do Sul. Realizou pesquisa na área de eletrônica para elaboração de kit para estudo de compatibilidade eletromagnética. Assuntos de interesse: compatibilidade eletromagnética, eletrônica, programação. 